\title{
A Node Cooperative ARQ Scheme for Wireless Ad-Hoc Networks
}

\author{
Mehrdad Dianati, Xinhua Ling, Sagar Naik, and Xuemin Shen \\ Department of Electrical and Computer Engineering, \\ University of Waterloo, \\ Waterloo, Ontario, Canada N2L 3G1
}

\begin{abstract}
We propose a Node Cooperative Automatic Repeat reQuest (ARQ) scheme for wireless ad-hoc networks to combat the effects of channel fading on the performance of link layer retransmission scheme. The proposed scheme is inspired by the idea of cooperative diversity. Simulation results are given to demonstrate the significant throughput gain, even when the quality of channel between the communicating nodes is poor.
\end{abstract}

\section{Introduction}

It is well known that the average bit error rate over wireless channels is several orders of magnitude higher than that in wired or fiber optic channels. Automatic Repeat reQuest schemes (ARQ) are de facto parts of wireless link layer to combat channel unreliability and to avoid expensive retransmissions by the transport layer's error control mechanism [1][2]. The conventional ARQ schemes have been developed for wireline networks, where frame errors are random, i.e., there is no correlation between frame error probabilities for different frames. However, due to the inherent characteristics of the fading process in wireless channels, frame errors appear in bursts rather than randomly. When the link between two communicating nodes is experiencing frame errors, there is a high probability that the bad channel condition will persist for a period as long as the transmission time of multiple data frames. Conventional retransmission schemes are not very effective in such environments with bursty frame errors, and will cause significant degradation in the performance of the link layer. This is because the sender retransmits data frames by remaining oblivious to the fact that even the retransmitted packets will encounter bit errors with very high probability due to the persistence of the bad channel condition.

In this paper, we propose a simple, but efficient, ARQ scheme, namely the Node Cooperative Stop and Wait (NCSW) scheme. The proposed scheme is inspired by the idea of cooperative diversity to improve the performance of link layer protocols in wireless networks [3]. We compare the performance of the proposed NCSW scheme with the conventional retransmission scheme. Our simulation results show that cooperation of a small number of nodes can produce significant throughput gain, even when the average quality of the channel between the sender and the receiver nodes is poor.

The rest of this paper is organized as follows. The system model is specified in Section 2. The proposed ARQ scheme is explained in Section 3, and the simulation results are given in Section 4. Finally some concluding remarks are given in Section 5. 


\section{System Model}

We consider an ad-hoc wireless network model as shown in Fig. 1. For the sake of generality, a group of autonomous nodes without any central control are assumed. A single node in this model can be viewed as a mobile device, a base station or an access point. A cooperation group is a subset of nodes that can reach one another with a single hop. In other words, nodes in a cooperation group are in the radio coverage area of one another. Those groups may be set up during connection stage or link level handshaking (e.g., RTS/CTS in 802.11). A node may join several cooperation groups depending on its position, capability, and willingness to cooperate. As shown in Fig. 2, each of those cooperation groups can be modelled as a single hop wireless network. At any instant of time, one sender node captures the shared medium to send a burst of frames to its intended destination node. During that time period other nodes (i.e., neighbor nodes) in the group keep listening to the shared channel, and assist the sender and the receiver nodes if error happens.

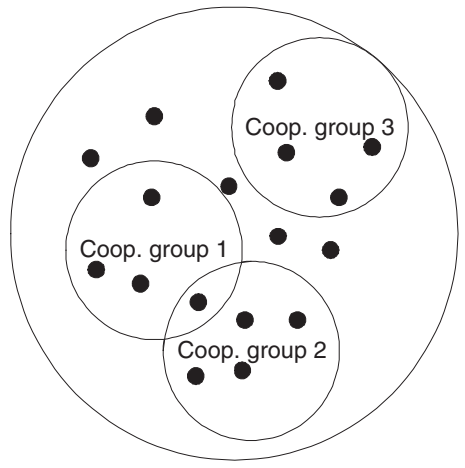

Fig. 1. An ad-hoc wireless network model

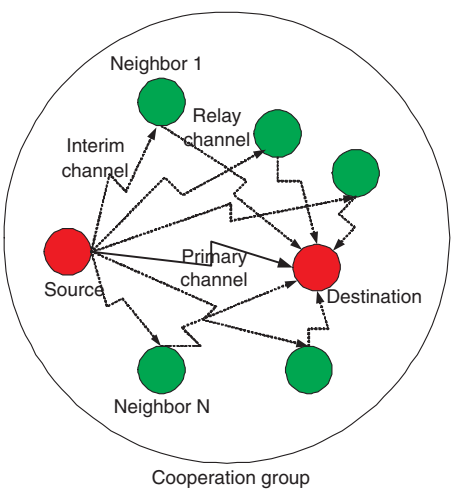

Fig. 2. A single cooperation group

Since long range wireless networks, such as satellite networks, are not considered in this paper, the propagation delays among communicating nodes are assumed to be relatively small (e.g., wireless LANs and cellular networks). In those systems, a Stop and Wait (SW) retransmission discipline is more appropriate than the Go Back N (GBN) and Selective Repeat (SR) schemes. In the SW scheme, the sender node does not transmit the next frame until the correct reception of the previous frame is confirmed by an explicit or implicit ACK. We assume that the reverse channel, used for ACK/NAK, is error-free. Thus the ACK/NAK frames can be received immediately and correctly by all the nodes in a cooperation group.

\section{Node Cooperative SW Scheme}

Depending on the relative velocity of communicating nodes, the duration of channel fading can be as long as the transmission time of several frames. For example, in a slow 
fading channel with a node speed of $5 \mathrm{Km} / \mathrm{h}$ and carrier frequency $f_{c}=2400 \mathrm{MHz}$, the average fading duration is about $60 \mathrm{~ms}$ [4]. For a typical frame length of $5 \mathrm{~ms}$, the conventional SW scheme has to retransmit the erroneous frame for an average of 12 times. The situation will be worse in high-rate systems with a shorter frame duration.

With the proposed NCSW ARQ scheme, the neighbor nodes in a cooperation group (Fig. 2) monitor the ongoing communication between a sender and a receiver node, decode the received frame, and store a copy of the most recently receicved frame. If the receiver can not decode a frame correctly, it will send a NAK to the sender. Consequently, the sender will respond by retransmitting the frame. In the SW scheme, the neighbor nodes are oblivious to the retransmissions. However, in the NCSW scheme, when the neighbor nodes in a cooperation group receive a NAK, they will transmit the requested frame to the receiver concurrently with the retransmission trials by the sender. When a frame is acknowledged by the receiver, all the nodes in the cooperation group drop their corresponding copy of the acknowledged frame. Obviously, a neighbor node in the cooperation group can retransmit only if it has already received a correct copy of the requested frame. Even if a neighbor node has a correct copy, cooperation can be optional. This guarantees the backward compatibility of the NCSW protocol with the conventional SW protocol.

\section{Simulation Results}

We simulate a single hop ad-hoc network with one pair of sender-receiver nodes and varying number of neighbor nodes, as shown in Fig. 2. The channels among the nodes are generated by the Rayleigh fading model. The carrier frequency is $2400 \mathrm{MHz}$ and the relative speed of the nodes is $5 \mathrm{Km} / \mathrm{h}$. The quality of the channels are represented in terms of the ratio of the fading margin and the mean value of the fading envelope, namely $L$. When the value of fading envelope is below the fading margin, the transmitted frame can not be decoded properly. As the value of the fading margin increases, poorer channel qualities and more frame errors are encountered. The data frame duration is assumed to be $5 \mathrm{~ms}$, which is a reasonable value for many wireless data networks. Perfect ACK/NAK information on the feedback channels is assumed to be available for the whole cooperation group right after a frame transmission. The numerical results are obtained from Monte-Carlo simulations.

To observe the impact of a small number of cooperative nodes on the system throughput, the SW and the NCSW schemes with only 2 neighbor nodes are simulated. The throughput of the both schemes are obtained by simulations. As shown in Fig. 3, the results are plotted against variations of the quality of the primary channel, as denoted by $L_{p}$ ( $L$ of the primary channel). The quality of all the interim and the relay channels are assumed to be identical, and denoted by $L_{r}$ ( $L$ of the relay channel). To demonstrate the impact of the variations in the quality of the interim and relay channels, throughput of the NCSW protocol is plotted for two different values of $L_{r}$, namely $-5 d B$ and $-1 d B$, respectively. The results in Fig. 3 show that with cooperation of only 2 neighbor nodes, throughput of the NCSW scheme can be improved by up to $30 \%$, depending on the quality of the interim and relay channels. 


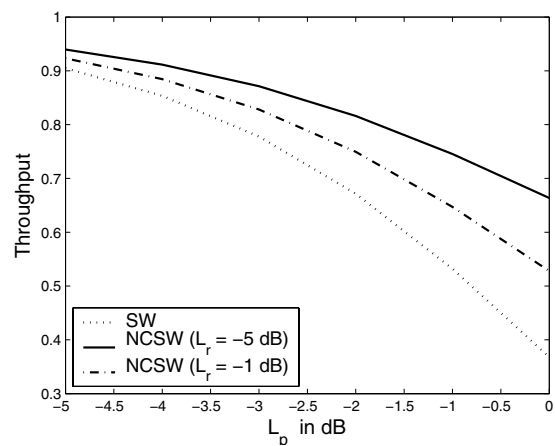

Fig. 3. Throughput vs. the fading margin

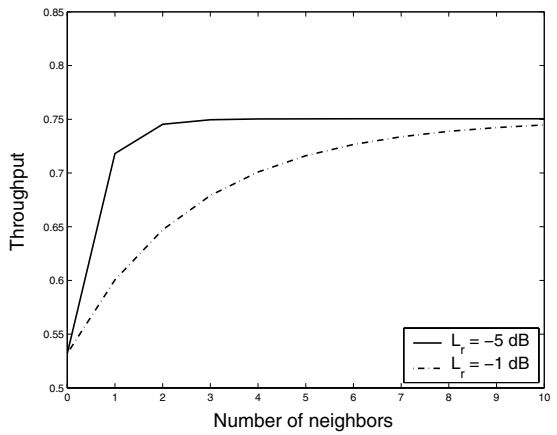

Fig. 4. Throughput vs. the number of neighbors

To observe the impact of the number of neighbor nodes on the protocol throughput, the fading margin of the primary channel is set to $L_{p}=-1 d B$. The simulations are performed for two different fading margins for the relay and interim channels, namely $L_{r}=-5 d B$ and $L_{r}=-1 d B$. As shown in Fig. 4, when the number of the cooperative nodes is increased, the system throughput approaches to a saturation level depending on the quality of the primary and the interim and relay channels. If the qualities of the interim and relay channels are good, having even one or two neighbor nodes can significantly improve the throughput. However, when the qualities of the interim and relay channels are poor, more neighbor nodes are required to achieve the same level of performance gain. Saturation in the system throughput is also expected. In fact, regardless of the number of neighbor nodes or their channel qualities, individual frame errors can not be prevented. However, cooperation of the neighbor nodes can reduce the impact of bursty erros.

\section{Conclusions}

In this paper, we have proposed a Node Cooperative Stop and Wait (NCSW) ARQ scheme for the wireless ad hoc networks. The simulation results demonstrate that the effectiveness of retransmission scheme, in terms of throughput, can be improved significantly by cooperation among the adjacent nodes in wireless ad-hoc networks.

\section{References}

1. O. Gurbuz and E. Ayanoglu, "A transparent ARQ scheme for broadband wireless access," Proc. IEEE WCNC'04, 2004, vol. 1, March 2004, pp. 423 - 429.

2. A.R. Parsad, Y. Shinohara, and K. Seki, "Performance of hybrid ARQ for IP packet transmission on fading channel," IEEE Trans. Veh. Technol., vol. 48, no. 3, May 1999, pp. 900-910.

3. A. Sendonaris, E. Erkip, and B. Aazhang, "User cooperation diversity, Part I: System description” IEEE Trans. Commun., vol. 51, no. 11, Nov. 2003, pp. 1927-1938.

4. G.L. Stuber, "Principles of Mobile Communication,”, Kluwer Academic Publishers, 2001. 